\title{
A tumour suppressor function of caspase-8?
}

\author{
RA Knight ${ }^{*, 1}$ and DL Vaux ${ }^{*, 2}$ \\ Cell Death and Differentiation (2008) 15, 1337-1338; doi:10.1038/cdd.2008.102
}

Every so often, research throws up something unexpected. Science being inherently conservative, these reports are usually treated with greater rigour at peer review, and journals are often reluctant to publish contentious papers that do not broadly conform to the established dogma, unless the data are wholly compelling and there is a detailed molecular mechanism. However, withholding potentially heretical material from the wider scientific community deprives it both of intellectual initiative and of the practical opportunity to refute or even substantiate the observations. As might be expected, the more introverted and obsessive referees will recommend major revision with a lot more data, if not outright rejection, whereas the more extrovert and polemical will be more in favour of publication. These papers therefore present Editors with a difficult decision.

The paper by David Wallach's group, published in this issue of CDD, ${ }^{1}$ falls within this category (and we therefore side with the extroverts). The paper provides evidence that, independently from its classical role in mediating apoptosis induced by death receptor ligation, caspase-8 is involved in the suppression of oncogenic transformation. There have been few hints in the literature of this possibility, especially from the total and conditional caspase- 8 deletion studies. Two groups have generated total caspase-8 knockouts, ${ }^{2,3}$ although in one, there was a residual catalytically inactive fragment. Both, however, broadly agree on the phenotype, with the most prominent features being embryonic lethality around 13$14 \mathrm{dpc}$ associated with marked hyperaemia and a depleted haematopoietic precursor pool, together with poorly developed ventricular walls and neural tube defects. Although caspase-8 is expressed widely in the E9.5-E12.5 embryo, these data suggest that caspase- 8 is required for the normal development of specific cell types, ${ }^{4}$ although the detailed mechanism remains unknown. As expected, caspase-8deficient fibroblasts are resistant to apoptosis induced by death receptor ligands (but not to death caused by agents such as etoposide or UV irradiation, which are death receptorindependent), although the transcription factors NF- $\mathrm{BB}$ and AP1 are activated normally after treatment with TNF or FasL.

Conditional deletion of caspase-8 in hepatocytes, endothelial and bone marrow cells similarly confers resistance to death receptor-mediated death of these cell types, and disrupted endothelial expression produces the same yolk sac and congestive abnormalities seen in the total knockout. ${ }^{5}$ In bone marrow cells, caspase-8 deletion results in the arrest of haematopoietic precursor development, and in particular the failure of myelomonocytic precursors to differentiate into macrophages. Although this suggested that caspase- 8 may have functions distinct from apoptosis, these data offered no support for a role of caspase-8 in suppressing transformation. The T-cell conditional knockout, ${ }^{6}$ however, gave an indirect hint, because these mice develop an age-dependent lethal lymphoproliferative and lymphoinfiltrative disorder, consistent with an earlier report ${ }^{7}$ that humans with germline mutations in caspase-8 also develop lymphadenopathy and splenomegally. An association between genetic deletion or silencing of caspase-8 in human neuroblastomas with amplification of the MYCN oncogene ${ }^{8,9}$ has also been reported.

It has also been noted that caspase-8-deficient MEFs have reduced mobility, which is restored by re-expression of caspase- 8 but not caspase-3. ${ }^{10}$ Caspase- 8 promotes mobility, at least in part, by calpain activation, and calpains are known to be involved in the regulation of the adhesion complex. ${ }^{11}$ Calpains have also been implicated in accurate chromosomal alignment at metaphase ${ }^{12}$ and there is some evidence that caspase-8-deficient MEFs are more prone to become multinucleated. ${ }^{10}$ It is rather a large leap to argue from here that caspase- 8 deficiency contributes to the chromosomal instability that is a hallmark of many tumours (and which may be implicated in transformation), ${ }^{13}$ but the ability of viral FLIP to induce transformation, ${ }^{14}$ and the elevated levels of cFLIP in some human cancers, ${ }^{15}$ might be due to inhibition of caspase-8-mediated calpain activation instead of inhibition of caspase-8-mediated apoptosis.

So how does the present Krelin et al. paper take the story forward? Wallach's group immortalised fibroblasts from wildtype (wt) and caspase-8-deficient mice by SV40 large T antigen, and tested their ability to form tumours in nude mice and colonies in soft agar at sequential passage numbers. They found that cells from the caspase-8-deficient animals formed tumours/colonies from about the 10th passage, whereas longer passage in culture was required for tumour/ colony formation by cells from the wt mice. In these experiments, which were replicated with several independent MEF isolates, a much lower proportion of wt MEFs produced tumours in vivo, and, in particular, formed soft agar colonies.

\footnotetext{
${ }^{1}$ MRC Toxicology Unit, Hodgkin Building, Lancaster Road, PO Box 138, Leicester LE1 9NH, UK and ${ }^{2}$ Department of Biochemistry, La Trobe University, Melbourne, Victoria 3086, Australia

${ }^{*}$ Corresponding authors: RA Knight, MRC Toxicology Unit, Hodgkin Building, Lancaster Road, PO Box 138, Leicester LE1 9NH, UK. Tel: + 442079052216 ; Fax: + 44207905 2301; E-mail: r.knight@ich.ucl.ac.uk or DL Vaux, Department of Biochemistry, La Trobe University, Melbourne, Victoria 3086, Australia. E-mail: d.vaux@latrobe.edu.au
} 
Clearly, the potential failure of the knockout cells to die in response to endogenous death ligands in the nude mice cannot explain the soft agar data, and an additional, nonapoptotic, function of caspase-8 in suppression of transformation must be invoked. Although it would be interesting to compare the tumorigenic capacity of primary and immortalised wt and caspase-8-deficient cells, primary MEFs are unlikely to survive for sufficient passages for a transformed phenotype to emerge before undergoing senescence. SV40 large $T$ antigen immortalises cells by acting on three principal targets, the chaperone hsc70, Rb and p53, resulting in the inhibition of the tumour suppressor activity of $\mathrm{Rb}$ and $\mathrm{p} 53,{ }^{16}$ and the action of caspase-8 deficiency in accelerating transformation would seem to lie downstream of these direct SV40 targets. However, the exact genetic (or epigenetic) mechanisms by which caspase-8 deficiency promotes transformation remain unknown.

Although caspase-8-deficient MEFs generate a larger number of soft agar colonies more rapidly than wt cells, an overwhelming proportion of both knockout and wt cells seeded into soft agar die, and do so at similar rates. As cell transformation occurred in both the caspase-8 wt and mutant cells, but the caspase- 8 mutants required less passages, loss of caspase- 8 is not sufficient for transformation, but either predisposes cells to further oncogenic mutations, or is complemented by later spontaneous oncogenic changes. Therefore, it may be possible to get some insight into the mechanism by gene profiling and proteomic approaches that compare colony-forming cells and non-colony-forming cells.
So here are well-controlled, reproducible observations imbuing an old friend with a new, important and unexpected function. The results are so surprising that the Editors felt that publication was justified, even though the data are limited to SV40 large T-transformed MEFs, and there remains a small possibility that the effects are due to sv129/C57Bl6 background differences in genes physically linked to the caspase- 8 locus, rather than caspase- 8 itself. In publishing the paper, we hope that it inspires as much excited debate among our readers as it has between Editors and referees. We look forward to publishing the results of experiments that address some of the unresolved questions raised by the paper in future issues of CDD.

1. Krelin Y et al. Cell Death Differ 2008; 15: 1350-1355 (this issue)

2. Varfolomeev EE et al. Immunity 1998; 9: 267-276.

3. Sakamaki S et al. Cell Death Differ 2002; 9: 1196-1206.

4. Kruidering M, Evan GI. IUBMB Life 2000; 50: 85-90.

5. Kang T-B et al. J Immunol 2004; 173: 2976-2984.

6. Salmena I, Hakem R. J Exp Med 2005; 202: 727-732.

7. Chun HJ et al. Nature 2002; 419: 395-399.

8. Teitz T et al. Nat Med 2000; 6: 529-535.

9. Stupack DG et al. Nature 2006; 439: 95-99.

10. Helfer B et al. Cancer Res 2006; 66: 4273-4278.

11. Carragher NO et al. Curr Biol 2003; 13: 1442-1450.

12. Honda S et al. J Biol Chem 2004; 279: 10615-10623.

13. Fujiwara T et al. Nature 2005; 437: 1043-1047.

14. Sun $Q$ et al. J Biol Chem 2003; 278: 52437-52435.

15. Ryu BK et al. J Pathol 2001; 194: 15-19.

16. Ahuja D et al. Oncogene 2005; 24: 7729-7745. 